\title{
Antibacterial Activity of Alchornea cordifolia (Christmas bush) Leaves Extract on Carbapenem Resistant Enterobacteriaceae Causing Multi-drug Resistant Systemic Infections
}

\author{
Henry Kwadwo Hackman ${ }^{1 *}$ Reuben Essel Arhin ${ }^{2}$ Bright Kojo Azumah ${ }^{2}$ \\ David Boateng $^{2} \quad$ Blessing Nwosu ${ }^{2}$ Mark Appenteng ${ }^{3}$ \\ 1.Accra Technical University, Faculty of Applied Sciences \\ Department of Medical Laboratory Technology, P. O. Box GP 561, Accra, Ghana \\ 2.Accra Technical University, Faculty of Applied Sciences \\ Department of Science Laboratory Technology, P. O. Box GP 561, Accra, Ghana \\ 3.Centre for Plant Medicine Research (CPMR), Mampong-Akuapim, Ghana
}

\begin{abstract}
Carbapenems have been used for the treatment of systemic infections caused by Enterobacteriaceae. However, recent studies suggested that some Enterobacteriaceae are producing carbapenemases, which has limited the treatment options for carbapenem-resistant Enterobacteriaceae. Some of the emerging carbapenemase resistant Enterobacteriaeae that are causing multi-drug resistant systemic infections include Escherichia coli, Klebsiellae pneumoniae, Providencia rettgeri, Proteus mirabili, Pantoea species, Citrobacter koseri and Acinetobacter baumanii. There is therefore the need for alternative treatment regimens for carbapenem-resistant Enterobacteriaceae. This study determined the in vitro efficacy of Alchornea cordifolia on carbapenem-resistant Enterobacteriaceae using agar well diffusion and well microplate dilution method. Serial dilutions of the ethanolic crude extract of the leaves were prepared and used against the carbapenem-resistant Enterobacteriaceae. The phytochemical screening was also performed to determine the antibacterial compounds. The christmas bush leaves extracts concentrations ranging from $50 \mathrm{mg} / \mathrm{ml}-200 \mathrm{mg} / \mathrm{ml}$ showed active diameter zone of inhibition. The ethanolic extract of Christmas bush leaves had minimum inhibition concentration and minimum bactericidal concentration of $3.13 \mathrm{mg} / \mathrm{ml}$ indicating significant antibiotic activity against the carbapenem-resistant Enterobacteriaceae. The phytochemical screening of the Christmas bush leaves showed the presence of antimicrobial phytochemicals such as flavonoids. This offers the possibility of developing effective antimicrobial agent to treat multi-drug resistant systemic infections. Therefore, there is the need to determine the toxicological effect and perform clinical trials of the active antimicrobial compounds isolated in the leave extracts of Christmas bush shrub.
\end{abstract}

Keywords: Alchornea cordifolia, Flavonoids, Antibacterial, Carbapenem-resistant Enterobacteriaceae

DOI: $10.7176 / \mathrm{JNSR} / 10-10-03$

Publication date:May $31^{\text {st }} 2020$

\subsection{Introduction}

Enterobacteriaceae are inhabitants of the intestinal flora and are the source of community and hospital acquired systemic infections. They have the propensity to spread easily between humans (hand carriage, contaminated food and water) and to acquire genetic material through horizontal gene transfer, mediated mostly by plasmids and transposons (Nordmann et al, 2011). Since 2000, spread of community acquired enterobacterial isolates that produce extended-spectrum $\beta$-lactamases (ESBLs) capable of hydrolyzing almost all cephalosporins except carbapenems has been reported worldwide (Pitout and Laupland, 2008).

There are strains of Enterobacteriaceae that can produce carbapenemase, an enzyme capable of destroying carbapenem antibiotics. Some of the emerging carbapenemase resistant Enterobacteriaeae (CRE) responsible for systemic infections include Escherichia coli, Klebsiellae pneumonia, Providencia rettgeri, Proteus mirabili, Pantoea species, Citrobacter koseri and Acinetobacter baumanii (Hackman et al., 2017). Systemic bacterial infections affect almost all part of the human body systems causing infections such as sepsis, pneumonia, peritonitis, gastroenteritis, meningitis and urinary tract infections.

Carbapenems are a valuable family of antibiotics normally reserved for serious systemic infections caused by drug resistant Gram-negative bacteria (including Enterobacteriaceae). They include meropenem, ertapenem, imipenem and doripenem. The presence of carbapenamase makes Enterobacteriaceae resistant to multiple antimicrobials and therefore infections caused by CPE (Carbapenamase producing Enterobacteriaceae) limit treatment options. The first carbapenemase producer in Enterobacteriaceae (NmcA) was identified in 1993 (Naas and Nordmann, 1994). Since then, a large variety of carbapenemases has been identified in Enterobacteriaceae belonging to 3 classes of $\beta$-lactamases (Queenan and Bush, 2007).

One of the major public health challenges in the 21st century is the increasing dominance of antibiotic resistant strains in most bacterial infections. Antibiotic resistance in bacteria may be an inherent character of the 
organism that renders it naturally non-susceptible to specific antibiotics. The misuse of antibiotics in humans and animals is accelerating the process of antibiotic resistance development. When antibiotics are misused or over prescribed, bacteria become resistant to their effects, making some infectious diseases difficult to treat.

Previous studies by Hackman et al., 2013 in Accra established that extended-spectrum beta-lactamase (ESBL) producers were increasingly becoming a public health nuisance due to community acquired and nosocomial infections. Since ESBL producers resulted in multi-drug resistance among most beta-lactams and non-beta-lactams, carbapenems such as imipenem, meropenem and ertapenems are often the last resort for the treatment of ESBL infections (Hackman et al., 2017). Until recently, carbapenems have been successfully used for the treatment of infections caused by Enterobacteriaceae, including those producing extended-spectrum beta-lactamases (Vardakas et al., 2012). However, carbapenemases confer resistance to broad-spectrum antibiotics, usually including carbapenems, and therefore, the majority of carbapenemase-producing Enterobacteriaceae are carbapenem resistant (CRE).

Antibiotic treatment options for these multidrug-resistant infections are limited (Falagas et al., 2013). The screening of natural products has been the source of innumerable therapeutic agents (Korosecchviz and HoweGrant, 1992). Higher plants, as a source for new potential drugs is still largely unexplored and only a small percentage of them has been subjected to phytochemical investigation and the fractions submitted to pharmacological screening is very low (Saraswathy et al., 2011).

The leaves, stems and roots of Alchornea cordifolia (Schumach. and Thonn.) Müll. Arg. are used as traditional medicine in many African countries for the management of gastrointestinal, respiratory and urinary tract infections as well as for the treatment of wounds (Siwe Noundou et al., 2016).

According to George et al., (2010), the potency of the A. cordifolia leaves extract could be attributed to various classes of compounds such as steroids, tri-terpenoids, phenols and polyphenols, alkaloids, flavonoids, anthraquinones, saponins and tannins, already known to be present in A. cordifolia leaves.

Siwe Noundou et al. (2016) isolated seven compounds from A. cordifolia with antibacterial activities namely stigmasterol, stigmasta-4,22-dien-3-one, friedelin, friedelane-3-one-28-al, 3- $O$-acetyl-aleuritolic acid , 3-Oacetyl-erythrodiol and methyl-3,4,5-trihydroxybenzoate (methyl gallate).

In this study, the antibacterial activity of ethanolic extract of the leaves of $A$. cordifolia was tested against carbapenem-resistant enterobacteriaceae by determining its minimum inhibition concentration, minimum bactericidal concentration and percentage inhibition concentration of $A$. cordifolia extract. The study was based on the hypothesis that $A$. cordifolia will possess phytochemical compounds that will have antimicrobial properties against Carbapenem-resistant enterobacteraeceae through in vitro analysis.

\subsection{Methods}

2.1 Study Site

This research work was carried out at the Centre for Plant Medicine Research, Mampong-Akuapem in the Eastern Region of Ghana.

\subsection{Sampling of Test Plant Material and Extracts Preparation}

Thousand gram $(1000 \mathrm{~g})$ of the plant leaves were collected and authenticated at the herbarium at the Centre for Plant Medicine Research (CPMR), Mampong-Akuapem. The sampling was based on the assumption that the plant is healthy and uninfected. The plant parts were cut into pieces, air dried at $26 \pm 2{ }^{\circ} \mathrm{C}$ for ten days and milled into coarse powder with the aid of an electric blender.

\subsection{Ethanolic Extraction, Evaporation and Freeze Drying of Extracts}

Three hundred grams $(300 \mathrm{~g})$ of the powdered plant part was soaked in $2 \mathrm{~L}$ of $96 \%$ absolute ethanol for 72 hours with occasional stirring and was filtered through Wathman's No.1 filter paper. The filtrate was concentrated with the aid of a rotary evaporator and was freeze-dried using the freeze drying machine at a temperature and reduced pressure of $35^{\circ} \mathrm{C}$ and $100-80 \mathrm{hPa}$ respectively. The dried extract was stored under refrigeration condition $\left(4^{\circ} \mathrm{C}\right)$ until needed.

\subsection{Reconstitution of Serial Concentrations of the Crude Extract}

Eight hundred milligrams $(800 \mathrm{mg})$ of the dried crude extracts were disolved in $4 \mathrm{ml}$ of $5 \% \mathrm{v} / \mathrm{v}$ dimethyl sulphoxide (DMSO) to prepare $200 \mathrm{mg} / \mathrm{ml}$ concentration of the leaves extract. Subsequent concentrations of 100, 50, 25, and $12.5 \mathrm{mg} / \mathrm{ml}$ were achieved using the conventional two folds dilution technique.

\subsection{Preparation of Muellar Hinton Agar (MHA) as a Culture Media}

The culture media were prepared according to the manufacturers instruction. Thirty eight grams $(38 \mathrm{~g})$ of the powder was suspended in $1000 \mathrm{ml}$ of demineralized water and heated to boil with agitation to completely dissolve the powder. The medium was then sterilized by autoclaving at $121^{\circ} \mathrm{C}$ for 15 minutes. 


\subsection{Determination of Carbapenem Resistant Enterobacteriaceae (CRE) Isolates}

The Kirby-Bauer method of antibiotic susceptibility testing was used to determine the susceptibility of the isolates to carbapenems (ertapenem, imipenem, meropenem). Etest system (bioMérieux, France) was also used to determine the minimum inhibition concentration (MIC) of the carbapenems. The therapeutic significance of the zones of inhibition and MICs was based on the Clinical and Laboratory Standards Institute (CLSI) breakpoints. Bacterial isolates that were resistant to the carbapenems were considered carbapenem resistant enterobacteriaceae (CRE) isolates.

\subsection{Subculturing of Carbapenem Resistant Enterobacteriaceae (CRE) Isolates}

The test organism was subcultured onto Mueller Hinton agar plate and was incubated for 24 hours to obtain a pure culture and to ascertain the viability of the organism. At least four colonies from the pure culture were transfer into sterile peptone water using an inoculation needle and adjusted to turbidity equivalent to $0.5 \mathrm{McFarland}$ Standard.

\subsection{Antimicrobial Susceptibility Testing Using Agar Well-Diffusion Method}

Antimicrobial activity of the plant extracts was assessed using the agar- well diffusion method. A $3 \mathrm{ml}$ inoculum was prepared in peptone water and adjusted to $0.5 \mathrm{McF}$ arland and uniformly spread on the MHA plate using the glass spreader. Wells $(6 \mathrm{~mm}$ diameter) were created in the plate using cork borer and $20 \mu 1$ of the plant extract concentrations $(200 \mathrm{mg} / \mathrm{ml}-12.5 \mathrm{mg} / \mathrm{ml})$ were pipetted into the wells at their respective concentration. Amikacin $(30 \mu \mathrm{g} / \mathrm{ml})$ and colistin $(10 \mu \mathrm{g} / \mathrm{ml})$ disks were used as positive controls and 5\% v/v DMSO and sterile distilled water (SDW) were used as the negative controls. They were allowed to diffuse at room temperature for 30 minutes and the plates were then incubated at $37^{\circ} \mathrm{C}$ for 24 hours. All experiments were carried out in triplicate.

After incubation, the diameter zone of inhibition (DZI) were measured and expressed in millimeters. DZI $<7 \mathrm{~mm}$ was considered inactive; 7-10 mm partially active; 11-16 active and $>16$ very active as described by Junior and Zanil (2000).

\subsection{Determination of Minimum Inhibition Concentration (MIC)}

Hundred microlitres $(100 \mu \mathrm{l})$ of ethanol extract of the active plants were added to $100 \mu 1$ of $5 \%$ DMSO in the first well of the 96-well microplate and mixed well with a micropipette; $100 \mu$ of this dilution was transferred to the next well in the column. This two-fold serial dilution was continued to obtain dilutions ranging from $10^{1}$ to $10^{10}$ with the aid of a micropipette. The final volume in each well was $100 \mu$ l. Solutions of a reference antibiotic amikacin $(30 \mu \mathrm{g} / \mathrm{ml})$ and colistin $(10 \mu \mathrm{g} / \mathrm{ml})$ were also serially diluted in different columns of the microplate as a positive control. The wells were inoculated with $5 \mu \mathrm{l}$ of microbial suspension of turbidity equivalent to 0.5 $\mathrm{McF}$ arland and incubated at $37^{\circ} \mathrm{C}$ for 24 hours. After incubation, $40 \mu \mathrm{l}$ of $0.2 \mathrm{mg} / \mathrm{ml} \mathrm{INT}$ (p-Iodonitrotetrazolium violet) dissolved in sterile distilled water was added to each of the wells. The microplates were examined after additional 30 to 120 minutes of incubation. Bacterial growth was indicated by a red colour of INT reduced to the formazan. The lowest concentration at which a decreased in the red colour is apparent compared to the next dilution was taken as the MIC value.

\subsection{Determination of Minimum Bactericidal Concentration (MBC)}

The MBC values were deduced from those wells with lowest concentrations at which no growth took place after culture for 24 hours of incubation. A small sample from each of those wells was transferred to fresh nutrient agar plates and was incubated overnight at $37^{\circ} \mathrm{C}$ and plates were examined for the presence or absence of living organisms. Plates with no microbial growth were regarded as the minimum bactericidal concentrations.

\subsection{Phytochemical Screening}

Phytochemical screening methods described by Fong et al., (1977) and Ciulei (1982) were used to screen the various extracts for the presence of saponins, reducing compounds, polyuronides, phenolic compounds, alkaloids, triterpenes, phytosterols, flavonoids and anthracenosides.

\subsection{Results}

3.1 Susceptibility of Carbapenem-Resistant Enterobacteriaceae against Ethanolic Extract of Alchornea cordifolia

The ethanolic extract of Christmas bush leaves varied zones of inhibition as shown in Table 1: 
Table 1: Susceptibility of carbapenem-resistant Enterobacteriaceae to ethanolic extract of Alchornea cordifolia Standards Diameter of zones of inhibition (mm)

\begin{tabular}{llllllll}
\hline $\begin{array}{l}\text { Amikacin } \\
(30 \mu \mathrm{g} / \mathrm{ml})\end{array}$ & $\begin{array}{l}\text { Colistin } \\
(10 \mu \mathrm{g} / \mathrm{m})\end{array}$ & $\begin{array}{l}\text { SDW } / \\
\text { DMSO }\end{array}$ & $200 \mathrm{mg} / \mathrm{ml}$ & $100 \mathrm{mg} / \mathrm{ml}$ & $50 \mathrm{mg} / \mathrm{m}$ & $25 \mathrm{mg} / \mathrm{m}$ & $12.5 \mathrm{mg} / \mathrm{ml}$ \\
$22.0 \pm 0.5$ & $17.0 \pm 0.5$ & 0.0 & $16.3 \pm 1.0$ & $13.7 \pm 0.5$ & $11.9 \pm 1.3$ & $8.5 \pm 2.5$ & $0.0 \pm 0.0$
\end{tabular}

Values are mean inhibition zone $(\mathrm{mm}) \pm \mathrm{S} . \mathrm{D}$ of three replicates

3.2 Minimum Inhibition Concentration (MIC) and Minimum Bactericidal Concentration (MBC)

The minimum inhibition concentration (MIC) and minimum bactericidal concentration (MBC) shown in Table 2 were determined using the 96 well microplate.

Table 2: MIC and MBC of ethanol leaves extracts of Alchornea cordifolia against CREs

\begin{tabular}{lll} 
Plant extract & MIC $(\mathbf{m g} / \mathbf{m l})$ & MBC $(\mathbf{m g} / \mathbf{m l})$ \\
\hline Alchornea cordifolia & 3.13 & 3.13
\end{tabular}

\subsection{Phytochemical Constituents}

The phytochemical constituents of the $A$. cordifolia leaves extract were saponins, phenolic compounds, reducing sugars, polyuronoids and flavonoids as shown in Table 3. Previous work by Siwe Noundou et al., (2016) had identified the antibacterial compounds in $A$. cordifolia leaves and elucidated their chemical structure.

Table 3: Phytochemical constituents of $A$. cordifolia leaves

\begin{tabular}{ll}
\hline Phytochemical & A. cordifolia \\
\hline Saponins & + \\
Phenolic compounds & - \\
Reducing sugars & + \\
Polyuronoids & - \\
Alkaloids & - \\
Triterpenes & - \\
Phytosterols & - \\
Flavonoids & + \\
Anthracenosides & - \\
\hline
\end{tabular}

Detected $=(+)$; Not Detected $=(-)$

\subsection{Discussion}

The varied concentrations $(50 \mathrm{mg} / \mathrm{ml}-200 \mathrm{mg} / \mathrm{ml}$ ) of the Christmas bush leaves extracts, which were used against the CRE strain employing the agar diffusion method, revealed significant antibacterial activity against the test organism as shown in Table 2. Furthermore, ethanolic extracts of the shrubs' leaves had MIC and MBC of 3.13 $\mathrm{mg} / \mathrm{ml}$ against CREs.

This may be because Alchornea cordifolia leaves possess phytochemicals such as saponins, reducing sugars and flavonoids (George et al., 2010). Flavonoids have been known to possess the antibacterial effect. According to Siwe Noundou et al., (2016) antibacterial compounds such as stigmasterol, stigmasta-4,22-dien-3-one, friedelin, friedelane-3-one-28-al, 3-O-acetyl-aleuritolic acid , 3- $O$-acetyl-erythrodiol and methyl-3,4,5-trihydroxybenzoate (methyl gallate)were isolated from Christmas bush. It could be that the extract used in this study may contain these compounds since they fall under flavonoids and the phytochemical screening of the test plant revealed that it contains flavonoids as shown in Table 1 . There might be other phytochemicals that were not determined in this study, which may be responsible for the antibacterial activity of the $A$. cordifolia leaves.

The activity of the standards (amikacin and colistin) used in this study as positive control corresponds with the results of Hackman et al., (2017) although amikacin $(30 \mu \mathrm{g} / \mathrm{ml})$ used in this study shows a higher diameter zone of inhibition. According to Junior and Zanil (2000) diameter zone of inhibition of 11-16 is considered active and $>16$ is considered very active. From the results, concentrations of the test plant ranging from $50 \mathrm{mg} / \mathrm{ml}-200$ $\mathrm{mg} / \mathrm{ml}$ showed active diameter zone of inhibition. From Table 2, A. cordifolia had an MIC of $3.13 \mathrm{mg} / \mathrm{ml} \mathrm{with}$ $\mathrm{MBC}$ of $3,13 \mathrm{mg} / \mathrm{ml}$ with percentage inhibition index of 2.94 . This showed that the appropriate concentration of the ethanolic extract of Christmas bush leaves is able to inhibit the growth of the CREs.

\subsection{Conclusions}

The hypothesis for this study proved positive since the shrub under study showed activity against CREs. Ethanolic extracts of $A$. cordifolia leaves is proving to be efficacious against multi-drug resistant CRE, which is a major cause of serious systemic infections. Phytochemical screening of the leaves showed that it possesses flavonoids, saponins and reducing sugars, which is responsible for the antibacterial efficacy of the leaves extracts. This offers 
hope for the development of effective antibiotics for the treatment of multi-drug resistant systemic infections. Therefore, there is the need to determine the toxicological effect and perform clinical trials of the active antimicrobial compounds isolated in the leaves extracts of $A$. cordifolia.

\section{Abbreviations}

A. cordifolia: Alchornea cordifolia

CPE: Carbapenamase producing Enterobacteriaceae

CRE: Carbapenamase Resistant Enterobacteriaceae

CPMR: Centre for Plant Medicine Research

CLSI: Clinical and Laboratory Standards Institute

DMSO: Dimethyl Sulphoxide

DZI: Diameter Zone of Inhibition

E. coli: Escherichia coli

ESBL: Extended-spectrum beta-lactamase

INT: p-Iodonitrotetrazolium violet

MIC: Minimum Inhibitory Concentration

MBC: Minimum Bactericidal Concentration

MHA: Muellar Hinton Agar

SDW: Sterile Distilled Water

\section{Declarations}

Funding

Not Applicable

Competing interests

The authors declare that they have no competing interests.

Ethics Approval and consent to participate

Not applicable

Consent for publication

Not applicable

Availability of data and material

All data generated or analyzed during this study are included in this published article.

Authors' contributions

HKH designed the study. DB, BN, MA carried out the

experiments and collected the data.

$\mathrm{HKH}, \mathrm{BKA}$ and REA interpreted the clinical data. All authors' read and approved the

final version of the manuscript.

\section{Acknowledgment}

The authors acknowledge the logistical support by Centre for Plant Medicine Research.

\section{References}

Ciulei I (1982) Methodology for analysis of vegetable drugs. Practical Manuals on Industrial Utilization of Medicinal and Aromatic plants. Edited by the Ministry of Chemical Industry, Bucharest pp 73

Falagas ME, Lourida P, Poulikakos P, Rafailidis PI, Tansarli GS (2013) Antibiotic Treatment of Infections Due to Carbapenem-Resistant Enterobacteriaceae: Systematic Evaluation of the Available Evidence. Antimicrobial Agents and Chemother 8: 654.

Fong EHS, Tin-Wa M, Farnworth NR, Dobberstein RH (1977) Phytochemical Screening Methods. Department of Pharmacognosy and Pharmacology. College of Pharmacy, University of Illinois, USA

George NJ, Obot IB, Akot AN, Apkan AE, Obi-Egbedi NO (2010) Phytochemical and antimicrobial properties of leaves of Alchornea cordifolia. E-J. Chem. 7(3):1071-1079

Hackman HK, Osei-Adjei G, Ameko E, Kutsanedzie F, Gordon A, Laryea E, Quaye S, Anison L, Brown CA, Twum-Danso K (2013) Phenotypic Determination and Antimicrobial Resistance Profile of Extended Spectrum Beta-lactamases in Escherichia coli and Klebsiella pneumoniae in Accra, Ghana. Journal of Natural Sciences Res 3(12): 75

Hackman KH, Arhin ER, Gordon A, Mensah BNS (2017) Emergence of Carbapenem-resistant Enterobacteriaceae among Extended-spectrum Beta-lactamase Producers in Accra, Ghana. Journal of Natural Sciences Res 7: 1, 4-5.

Junior A, Zanil C (2000) Biological screening of Brazilian medicinal plants. Brazilian Journal of Sc 5

Korosecchviz I, Howe-Grant M (1992) Kirk-othmer Encyclopedia of Chemical Technology pp 893. 
Naas T, Nordmann P (1994) Analysis of a carbapenem-hydrolyzing class A $\beta$-lactamase from Enterobacter cloacae and its LysR-type regulatory protein. Proc Natl Acad Sci 91

Nordmann P, Naas T, Laurent P (2011) Global spread of carbapenemase-producing Enterobacteriaceae. Emerging infectious Dis 7: 1791

Pitout JD, Laupland KB (2008) Extended-spectrum $\beta$-lactamase-producing Enterobacteriaceae: an emerging public-health concern. Lancet Infect Dis 159.

Queenan AM, Bush K (2007) Carbapenemases: the versatile $\beta$-lactamases. Clin. Microbiol Rev 20.

Saraswathy A, Gunalan G, Krishnamurthy V (2011) Antimicrobial Activity of Medicinal Plant Bauhinia variegate Linn. International Journal of Pharmacy and Biological Sc 1:400.

Siwe Noundou X, Krause RWM, van Vuuren SF, Ndinteh DT, Olivier DK (2016) Antibacterial effects of Alchornea cordifolia (Schumach. \& Thonn.) Müll. Arg extracts and compounds on gastrointestinal, skin, respiratory and urinary tract pathogens. $J$ of Ethnopharmacology 179:76-82

Vardakas KZ, Tansarli GS, Rafailidis PI, Falagas ME (2012) Carbapenems versus alternative antibiotics for the treatment of bacteraemia due to Enterobacteriaceae producing extended-spectrum beta-lactamases: a systematic review and meta-analysis. J. Antimicrob. Chemother 67:2793-2803. 